\title{
Alignment of Shared Forests for Bilingual Corpora
}

\author{
Adam Meyers, Roman Yangarber, Ralph Grishman \\ New York University \\ 715 Broadway, 7th liloor, NY, NY 10003, USA \\ meyers/roman/grishman@cs . nyu .edu
}

\begin{abstract}
Research in example-bascd machine translation (TIBM'I) has been hampercd by the lack of efficient tree alignment algorithms for bilingual corpora. This paper clescribes an alignment algorithm for EBMT whose ruming time is quadratic in the size of the input parse trees. The algorithm uses dynamic programming to score all possible matching nodes between structure-sharing trees or forests. We describe the algorithm, various optimizations, and our implementation.
\end{abstract}

\section{Introduction}

T'he development of a machine translation (MT') system requires the lengthy manual preparation of bilingual lexicons and transfor rules. Research over the past fow years using parallel sentencealigned bilingual corpora suggests ways in which this manual effort can be partly replaced by corpus-based training. Some of this research has treated the sentences as unstructured word sequences to be aligned; this work has primarily involved the acquisition of bilingual lexical correspondences (Chen, 1993), although there has also been an attempt to create a full M'I system based on such treatment (Brown et al., 1993). Recently, several groups have been exploring the possibility of aligning parallel syntactically analyzed scntences from the source and target languages (cf. (Sato and Nagao, 1990), (Klavans and Tyoukemann, 1990), (Grishman and Kosaka, 1992), (Kaji ct al., 1992), (Matsumoto et al., 1993) and (Grishman, 1994)). 'Ihis offers the potential for acquiring not just lexical but also structural correspondences between the two languages. The specific goal in aligning syntax trees is to identify the corresponding tree fragments in the source and target trees. By processing a substantial corpus, a large set of such corresponding fragments can be collected. 'These can then serve as the example base for a form of cxample-based M'I' (cf. (Nagao, 1984), (Sato and Nagao, 1990), (Kaji et al., 1992), (Matsumoto ot al., 1993) and (luruse and lida, 1994)). 'This approach requires a fast tree alignment technique; research has been hampered by the lack of officiont algorithms. 'This paper describes an efficient algorithm for bilingual tree alignrment.

\section{Our Approach}

For each input sentence our parser produces a set of trees, corresponding to cach possible syntactic analysis. Our parse trees are transformed into a "regularized" format, to represent the PredicatioArgument structure. For each sentence, the output of the parser is a structure-sharing forest. An cxample of structure sharing between two parse trees of the same input sentence is shown in lighure 1 . We apply the parser to the soure and target sentences, using a Spanish and an Finglish grammar, respectively. 'The resulting sets of structuresharing parse trees form the input to the alignment procedure.

Our alignment program employs dynamic programming algorithms, which are clescribed in detail in later sections. 'The program begins al the roots of the source and target trees, and proceeds top-down recursively, filling a matrix of scores. Given $N$ nodes in the source tree $T_{s}^{\prime}=T\left(V_{s}, V_{s}\right)^{2}$ and $M$ nodes in the target tree $T_{t}=T\left(V_{l}, t_{t}^{\prime}\right)$, the score matrix is an $N \times M$ matrix. lor each pair of nodes $x_{i}, i=1, \ldots N \in V_{s}$ and $y_{j}, j=1, \ldots M \in V_{t}$, the corresponding entry in the score matrix is a measure of how woll $x_{i}$ matches $y_{j}$. 'The score for cach pair of nodes depends only on the closeness of the lexical cntries associated with the nodes and

\footnotetext{
${ }^{1}$ Cf. c.g. (Cormen et al., 1990), pp.299-328

2'The expression $T\left(V_{s}, h_{s}^{\prime}\right)$ clemotes a tree as a pair of sets: $V_{s}$ is the set of vertices (nodes) in the tree, and $E_{s}$ is the set of edges (ares).
} 


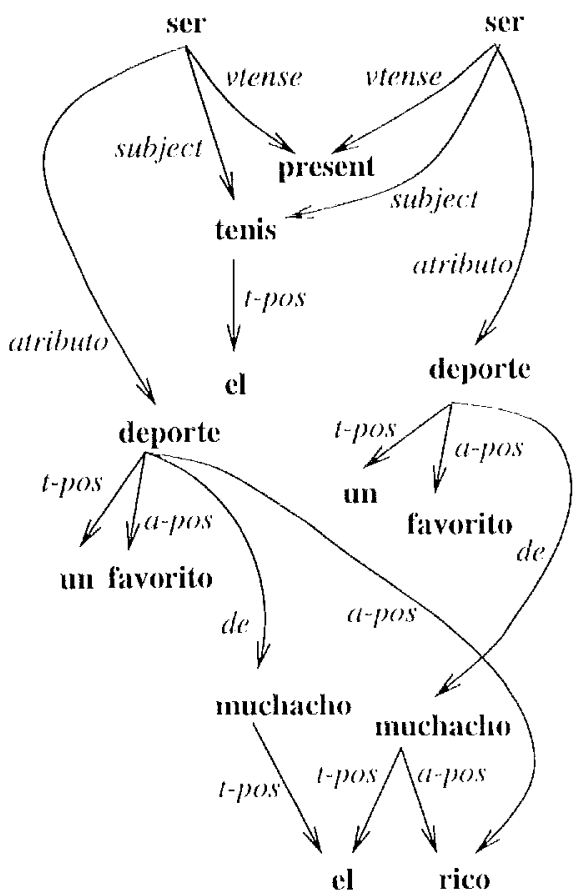

Figure 1: 'L'wo parses for: lil temis es un deporte? favorito del muchacho rico.

on the scores of the best matching pairs of their descendants. Dynamic: programuming assures that each entry in the materix, (i.e. hesesce for the cor responding pair of nodes) is computed only once.

Wo have implemented this approach for 185 sets tences from two soures: Lit Camino Real (a Spanish textbook from which we used 733 sentences with their linglish transtations), and (anous (iconge or forge d Curoso (the linglish and Spanish ver sions of II. A. Ray's popular children's book, from which we used all 1.11 linglish sentences and 112 Spanish Sentences). Of the totial 185 sentences, 57 from lil Camino Real and 55 from Curous Georye produced at least one parse tree in bolh languages. 'l'he aligmment proceduro was applied only to these palits of sentencess."

\section{Data Structures}

Regularized parses are similas to the listructume

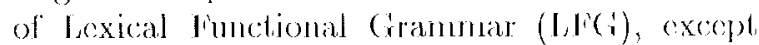
that a dependency type structure is assumed.

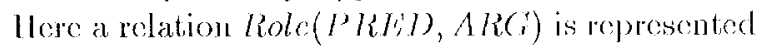

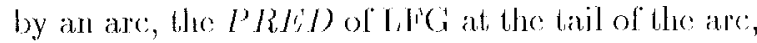
and the ARG (or the role recipient) at the head

\footnotetext{
Byy inproving the asymptotic speed of alignment on these few sentenees, we open the possibility for us ing much lasger corjogra in lubure work.

4(Sato and Nagao, 1.90) aud (Matisimoto ed al. 1993) also assinte dependency type structures in theji example-based work.
}

of the are. For example, ef tenis is the subject of the predicate ser in ligure 1. In a regular. ized parse, certain closed syntaketic classes, such as prepositions and subordinate conjunctions, are represented ats are labels denoting roles, (e.g., the preposition de in figne 1) rather than as nodes in listructiuse.

Strueture shitring anong the trees in the parse forest allows us bo reduce the mumber of conpluted scores. Wo compute the score for a given pair of subtrees only once, regardless of the number of trees which share these subtrees becanse the score of a pair of nodes depends only on the seores of their desendants (and not of their aneestors). Currently our parser records structure shariug only between NP's. lixperinents in which all com mon structure is shareel, as in ligure 1, suggest. that extending structure sharing to other types of modes would further inporove performance. 'This structure-sharing approach is based on previous work in optimizing leature Structure-based parsing. (Soe lor example, (Kartimen, 1985) and (Percira, 1985)).

\section{The LCA-Preserving Algorithms}

We limt discuss the format aspectis of the alignment problem and introduce tominology.

\subsection{The Maximal Tree Alignment Problem}

'The ofjective is to find a maximmm-scome corecespondence between nodes in a pair of trees. 'The statement of the problem of aligning two trees $\%$ s and $\%$, corresponds closely to that found in (Mat.. smmoto of al., 1993). Our algorithms are based on those presented in (Stecl and Warnow, 1993), (liarach et al., 1995b) and (1995a).

Wo say that. a node $x$ is a common ancestor of nodes a and $b$ in a tree ' $f$ if there exist pathes of longth $\geq 0$ from $x$ to $a$ and from $x$ to $b$. 'The feast common ancestior (lea) of two nodes $a$ and $b$ is the node, $x_{0}=\log (a,(a, b)$, such that

1. $x_{0}$ is a common ancestor of a and $b$, and

2. For any other common ancestor $x$ of $a$ and $b$, $x_{0}$ is a descendant of $x$.

An aligmment between two treos $T-(V, b)$ and $l^{\prime \prime}=:\left(V^{\prime}, l_{f}^{\prime \prime}\right)$ is a correspondence (a one-to-one mapping) $f: S^{\prime}+S^{\prime \prime}$, where $S \subseteq V$ and $S^{\prime \prime} \subseteq V^{\prime}$, which maintains the following reationship:

For simplicity of prosentiation we state the problem in tecme of allignment of teces. In practice we are usimg an optimiged variant of the algorithm, which aligns pairs of stutucture-sharing forostis. 


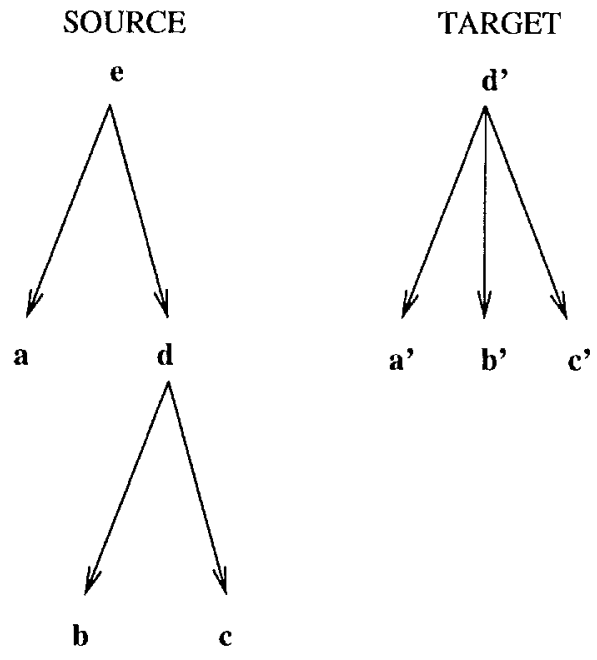

Figure 2: There is no lca-preserving alignment between $a, b$ and $c$, and $a^{\prime}, b^{\prime}$ and $c^{\prime}$.

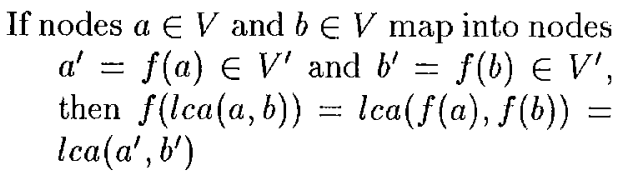

To illustrate, in Figure 2 there is no lca-preserving alignment of the two trees which maps all three of the leaf nodes $a, b$ and $c$ into the nodes $a^{\prime}, b^{\prime}$ and $c^{\prime}$. Lca-preserving alignments are possible which map any two of the leaves.

The algorithm assumes that least common ancestors are preserved in the alignment. We assign a score to each alignment based on the labels of the corresponding nodes and the arcs from these nodes, as described below. The algorithm seeks an alignment with maximal score.

\subsection{The Algorithm}

Let $T_{s}$ and $T_{t}$ be the source and the target trees. The algorithm uses dynamic programming to build up, in a bottom-up fashion, the scores for matching each node in $T_{s}$ against each node of $T_{t}$. There are $O\left(n^{2}\right)$ such scores, where $n=\max \left(\left|T_{s}\right|,\left|T_{t}\right|\right)$ is the number of nodes in the trees. Let $d(v)$ be the degree of a node $v$. We denote children of $v$ by $v_{i}, i=1, \ldots, d(v)$, and the $\operatorname{arc}\left(v, v_{i}\right)$ by $\vec{v}_{i}$.

Procedure SCORE $L C A$ : The dynamic programming builds up a score function $S\left(v, v^{\prime}\right)$ for all $v \in T_{s}$ and $v^{\prime} \in T_{t}$, which is stored in a $\left|T_{s}\right| \times\left|T_{t}\right|$ matrix $S$. The value $S\left(v, v^{\prime}\right)$ is the score assigned to the best match between the two subtrees rooted at $v$ in $T_{s}$ and at $v^{\prime}$ in $T_{t}$. Initially, $S$ is filled with undefined values. When a value for $S\left(v, v^{\prime}\right)$ is required, and the corresponding entry in the matrix is undefined, it is recursively computed by the following formula:

$$
S\left(v, v^{\prime}\right)=\max \left\{\begin{array}{l}
M A T C H_{l c a}\left(v, v^{\prime}\right) \\
\max _{i=1, \ldots, d(v)} S\left(v_{i}, v^{\prime}\right)-P\left(\vec{v}_{i}\right) \\
\max _{j=1, \ldots, d\left(v^{\prime}\right)} S\left(v, v_{j}^{\prime}\right)-P\left(\vec{v}_{j}^{\prime}\right)
\end{array}\right.
$$

The function $M A T C H_{l c a}\left(v, v^{\prime}\right)$ is a measure of how well the nodes $v$ and $v^{\prime}$ align, and is computed as follows:

$$
\begin{aligned}
& M A T C H_{l c a}\left(v, v^{\prime}\right)=\operatorname{Lex}_{n o d e}\left(v, v^{\prime}\right)+ \\
& \quad+\max _{p \in \mathcal{P}\left(v, v^{\prime}\right)} \sum_{(i, j) \in p} \operatorname{Lex}_{a r c}\left(\vec{v}_{i}, \vec{v}_{j}^{\prime}\right)+ \\
& \quad+S\left(v_{i}, v_{j}^{\prime}\right)
\end{aligned}
$$

where:

- Lex $x_{\text {node }}\left(v, v^{\prime}\right) \geq 0$ is a measure of how closely the label on source node $v$ corresponds to the label on target node $v^{\prime}$ in the bilingual dictionary. Lex $x_{\text {arc }}\left(\vec{v}, \vec{v}^{t}\right)$ is the corresponding rreasure for arcs.

- $\mathcal{P}\left(v, v^{\prime}\right)$ is the set of all possible pairings of the children of $v$ against the children of $v^{\prime}$. There are $O(d !)$ such pairings, where $d$ is the smaller of the degrees of $v$ and $v^{\prime}$.

- $P\left(\vec{v}_{i}\right) \geq 0$ is the penalty for collapsing the edge $\vec{v}_{i}$, which may depend on the label of that edge.

The summation in (2) ranges over all pairs, denoted by $(i, j)$, which appear in a given pairing $p \in \mathcal{P}\left(v, v^{\prime}\right)$. The summation is evaluated for all $O(d !)$ possible pairings. The pairing with the maximum score is then selected.

The total running time for computing the scores of all of the $O\left(n^{2}\right)$ node pairs $v$ and $v^{\prime}$, is $O\left(d ! n^{2}\right)$, where $d$ is the lesser of the degrees of the source and target trees. Computing the max term in (2) can be mapped into the Maximum-Weight Clique problem (which is NP-complete), cf. (Farach ct al., 1995b). However, in the NLP domain, the running time is contained because $d<6$ for most trees encountered in practice. Next we describe a heuristic which achieves a time bound quadratic in the size of the tree.

\section{A Greedy Heuristic}

We can reduce the computation time of the max term in (2), if we do not consider all of the $O(d !)$ pairings of the children of $v$ and $v^{\prime}$. Instead we 
use a greedy approach and choose the $d$ highestscoring, mutually disjoint pairs from among the $d^{2}$ possible pairs of children of $v$ and $v^{\prime}$. The justification for this heuristic is that we expect that the high-scoring pairs will dorninate, and will be $a$ priori mutually disjoint.

The following is an alternative, greedy procedurc for computing $S\left(v, v^{\prime}\right)$ :

\section{Procedure GREEDY $Y_{L C A}$ :}

1. $\forall i, j$ s.t. $1 \leq i \leq d(v), 1 \leq j \leq d\left(v^{\prime}\right)$ com pute the corresponding entry in a $d(v) \times d\left(v^{\prime}\right)$ matrix $M$ :

$$
M_{i j}=\operatorname{Lex} x_{a r c}\left(\vec{v}_{i}, \vec{v}_{j}^{\prime}\right)+S\left(v_{i}, v_{j}^{\prime}\right)
$$

The entry $M_{i j}$ of $M=M\left(v, v^{\prime}\right)$ is the score of matching the $i$ th child of $v$ with $j$ th child of $v^{\prime} .{ }^{6}$

2. Let $\mathrm{IOP}^{\prime} \leftarrow\{\}$ be the set of highest scoring pairs.

3. Find the largest entry $M_{i_{0} j_{0}}$ in the matrix, such that neither its row nor its column is already occupied by some pair in TOP:

$$
T O P \leftarrow T O P \cup\left\{\left(i_{0}, j_{0}\right)\right\}
$$

where the coordinates $\left(i_{0}, j_{0}\right)$ are such that

$$
\begin{aligned}
& M_{i_{0} j_{0}}=\max _{i, j}\left\{M_{i j}\right. \\
& \left.\quad \mid \forall\left(i^{\prime}, j^{\prime}\right) \in I^{\prime} O P, i \neq i^{\prime}, j \neq j^{\prime}\right\}
\end{aligned}
$$

1. Repeat the above step $d$ times, where $d=$ $\min \left(d(v), d\left(v^{\prime}\right)\right)$.

5. Compute the result:

$$
\begin{aligned}
& M A^{\prime} T^{\prime} C H_{l c a}\left(v, v^{\prime}\right)= \\
& =\operatorname{Lex} x_{n o d e}\left(v, v^{\prime}\right)+\sum_{(i, j) \in^{\prime} O P} M_{i j}
\end{aligned}
$$

With sorting, this can be done in $O\left(d \log d+d^{2}\right)$ time.

The validity of this heuristic can be tested by comparing the performance of the procedures using the computation in (2) and in (4).

\footnotetext{
${ }^{6}$ Note: if we disregard the are labels for simplicity, and set $\operatorname{Lex}_{\text {arc }}(\cdot, \cdot)=0$, then we do not need to build $M$, and may simply use $M_{i j}=S\left(v_{i}, v_{j}^{\prime}\right)$.
}

\section{Strict Lexical Matching Heuristic}

(Grishman, 1994) cmployed an optimization heuristic which favored lexical matches. For each source node $v$ with label $I(v)$, the procedure using this heuristic would first attempt to find a target node $v^{\prime}$ with label $L\left(v^{\prime}\right)$ such that $L(v)$ translated as $L\left(v^{\prime}\right)$ in the bilingual dictionary (a perfect lexical match). If such a lexical match was found, the procedure did not attempt to match $v$ with any other target node.

A similar heuristic (Lex-Match) was incorporated into our program as the following preprocessing steps:

1 For each source node $v$, all possible lexical matches are iclentified in the target tree. ${ }^{7}$ If $v$ has at least one possible lexical match, all of those positions in the score matrix $S$ which do not correspond to a lexical match of $v$ are set to yero.

2 For cach target node $v^{\prime}$ which has at least onc lexical match, all of those positions in the score matrix which do not correspond to a lexical match of $v^{\prime}$ are set to zero.

By setting to zero those positions in the score matrix which represent unlikely matches, this heuristic provents these scorcs from ever being calculated, substantially reducing the running time. I.ex-Match, unlike the (Grishman, 1994) heuristic, allows one source node to match lexically with more than one node in the target tree.

\section{Implementation}

We have implemented the greedy LCA-preserving algorithm with the following features:

Penalties: The penalties for collapsing edges were set to $0 .^{8}$

Scores: A lex node score of 100 and a lexare score of 21 was awarded for each match using our bilingual dictionary. 'These functions have the value 0 if there is no lexical match.

${ }^{7} \Lambda$ match $M\left(v, v^{\prime}\right)$ is also a lexical match if cither $M\left(v, w^{\prime}\right)$ or $M\left(u, v^{\prime}\right)$ is a lexical match, where $w$ and $w^{\prime}$ are children of $v$ and $v^{\prime}$, respectively.

${ }^{8}$ When penalties are set, to zero and an empty bilingual diclionary is used, the alignment algorithm fills the scoring matrix with zeros. When we introduce? non-zero penalties, the alignment procedure prefers matches betwcen nodes dominating similar structures, since nodes dominating dissimilar structures reccive negative scores. Wo expect that non-zero penaltics will improve precision with a nonempty bilingual dictionary, because they will favor similar structures. In preliminary testing, penalty values of 20 and 30 yielded improvements in precision. 


\begin{tabular}{|l|c|c|c|c|}
\hline Text & Baseline & Struc-Share & Lex-Match & Struc-Share and Lex-Match \\
\hline Tl Camino Real & $11.5 \mathrm{sec}$ & $11.3 \mathrm{sec}$ & $8.3 \mathrm{sec}$ & $7.7 \mathrm{sec}$ \\
\hline Curious George & $98.0 \mathrm{sec}$ & $18.8 \mathrm{sec}$ & $87.4 \mathrm{sec}$ & $44.7 \mathrm{sec}$ \\
\hline Total & $109.5 \mathrm{sec}$ & $60.1 \mathrm{sec}$ & $95.7 \mathrm{sec}$ & $52.1 \mathrm{sec}$ \\
\hline
\end{tabular}

Table 1: T'ime Improvements Due to Optimizations

\begin{tabular}{|l|l|l|}
\hline Text & Lex-Match Off & Lex-Match On \\
\hline WI Camino Real & 47 out of $57(82 \%)$ & 47.5 out of $57(83 \%)$ \\
\hline Curious George & 44.6 out of $55(81 \%)$ & 44.6 out of $55(81 \%)$ \\
\hline Total & 91.6 out of $112(82 \%)$ & 92.1 out of $112(82 \%)$ \\
\hline
\end{tabular}

T'able 2: Changes in Accuracy due to Lex-Match Heuristic

Optimization Variables: We experimented with variants of the procedures which included Structure Sharing (Struc-Share) and the Lexical Match Optimization (Lex-Match), as well as with those that did not.

Table 1 shows the time consumed by our program to align sentences under different conditions. 'The baseline refers to our program without anly optimizations (which is at lcast 6 times faster than bofore using this algorithm.) The optimization variables have different effects on the differcont texts. We believe that structure sharing has a much stronger effect on Curious George than on $\mathrm{El}$ Camino Real because the former has longer sentences which produced more parses. The liexMatch optimization has a greater effect on $\mathrm{Ll}$ Camino Real than on Curious George because all of the words contained in El Camino Real are included in our bilingual dictionary, but only a small portion of the words in Curious George are included. We expect that as the size of our dictionary increases, the Lex-Match optimization will have a greater effect.

'The precision for cach aligned pair of sentencess is computed according to the formula:

$$
\frac{\mid \text { ResultSet } \cap \text { Answerkey| }}{\mid \text { Resull Set } \mid}
$$

where Resultset is the sel of source parses to which the alignment procedure assigned the highest score, and Answerkey is the set of best source parses as judged by one of the expcrimenters.? This precision measure was previously used in (Matsumoto et al., 1993) and (Grishman, 1994). Table 2 compares the procision of the alignment procedure with and without, the Lex-Match heuristic (structure sharing had no effect on the scores.) The slight increase in precision observed with the

\footnotetext{
${ }^{9}$ If there was no correct parse, the parses with the fowest errors were used for purposes of alignment.
}

Lex-Match optimization, may be an indication that we should raise the score for lexical matches of node labels.

\section{Results and Future Directions}

The current implementation aligns trees 63 times faster than our previous program (Grishman, 1994), with a $2.3 \%$ improvement in precision. ${ }^{10}$ Wo expect finc-tuning of the paranneters in our procedures to improve our performance. We expect to gain greater efficiency if all common nodes betwoen forests are shared, rather than just the NPs. Another efficiency improvenent will be achicved by factoring all ambiguity into the parso tree, as in (Matsumoto of al., 1993). In our current approach, disjunctions are represented only at the root level.

In order to improve the precision of alignment, we plan to experiment with varying the values of the lex functions and penalties in our scor. ing algorithm and expanding one bilingual dictionary. We will also experiment with the non greedy algorithm discussed above and a dominancepreserving algorithm (a less constrained version of the algorithm which we have onitted due to space limitations). In the dominance-preserving algorithm we relax the requirement of lca-preservation, and require the preservation of the dominance selationship between nodes:

$$
\begin{aligned}
& \text { If, for two nodes } a \in T \text { and } b \in T \text {, } a \\
& \text { dominatios } b \text { (denoted as } a \prec b \text { ), then } \\
& \text { for } f(a) \in T^{\prime \prime} \text { and } f(b) \in T^{\prime \prime}, f(a) \prec \\
& f(b) \text {. }
\end{aligned}
$$

The idea which makes it possible to align sen tences quickly is that we place restrictions on the ways in which we align the parse treos. Wo

\footnotetext{
${ }^{10}$ The dynamic programming algorithm accounts for an approximately $600 \%$ increase in speed of aligmment

a rough estimate since much of the program has been re-implemented.
} 


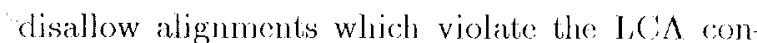
straint or the dominanes requirement, and permit only one-to-one aligmments between nodes. Some cases where one might posit a correspondence betwon a single node $x$ and a group of nodes $G=\left\{y_{1} \ldots y_{n}\right\}$, can be interpreted as ant alignment between $x$ and $y_{j}$, for somo $j, 1 \leq j \leq n$, where $y_{j}$ dominates the remaining nodes in 6 . We do not consider other types of one-to-many alignmonts.

\section{Acknowledgements}

Wo wish to thank Antonio Moreno Sandoval and Cristina Olmeda Moreno for preparation of the Spanish analyses for Jorge el Curioso. Wo also thank Catherine Macleod for preparation of the Finglish parses.

This rescarch was supported by the National Science Foundation under Grant IR.1-9303013.

\section{References}

Peter Brown, Stephen A. Della Pietra, Vincent, J. Della Pietra and Robert I. Moreer. 1993. 'The Mathematics of Statistical Machine 'I'ranslation: Parameter fistimation. In "Compulational Linguistics, 19: 263 312

S. Chen. 1993. Aligning Sontences in Bilingual Corpora using lexical juformation. In Procecelings of the 31st Annual Meeting of the Association for Computational linguestics, pages (9) 16 , Columbus Ohio. Association for Computational linguistics, Morristown, New Jersey.

'T. II. Commen, C. IS. Leiserson and R. I. Rivest. 1990. Introduction to Algorithms, 'The MT' Press, Cambridge, Mass.

Martin Warach, 'Toresa M. Pryytycka and Mikkel 'Thorup. 1995. Tho maximum agreemont subtree problem for binary tress. Unpublisted Ilanuscript, Rutgers University, Odense University, and Univorsity of Copenhagen.

Martin Parach, 'Icresa M. Pryytycka and Mikkel Ihorup. 1995. On the agreement of many trees. Unpublished mauuscript, Butgers Uni versity, Odense Iniversity, and University of (openhagen.

Osamu liuruse and llitoshi licla. 1994. Con stituent Bomdary Parsing for tixamplo-Based Machine "Transtation. In COJINC 9/ Proced ings, Volune 1, pages 105.111, Kyoto Japan.

Ratph Grishman. 1994. Iterative Aligmment of Syntactic: Structures for a Bilingual Corpus. In Procedings of the Second Anmual Workshoy for Very Large Corpora, Tokyo, Jajun.
Ralph (xrishman and Michiko Kosaka. 1992. Combining Rationalist and Fimpiricist $\Lambda_{p}$. proaches to Machine 'Translation. In Procectings of the lowrth International Conferenes on Theoretical and Molhodological Issues in Machine Transtation, Montroal, Canada.

Hiroyuki Kaji, Yuuko Kida and Yasututsugo Morimoto. 1992. Ioarning 'Tanslation 'Templates from Bilingual Text. In Coling go procedings.

Lauri Kartumon. 1985. Structure-Sharing with

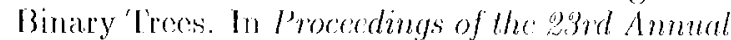
Mceling of the Assoriation for Compulational linguistics.

Judith Klavans and livelyne T'zoukermann. 1990.

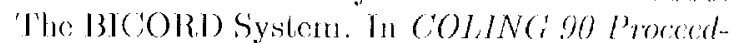
ings, Volume 3, panges 174179.

Y. Matsumoto, II. Ishimoto, T'. Utsuro and M. Nagia. 1998. Suructumal Matching of Parallel 'lexts. In 31st Anmual Meeting of the Associalion for Compulational Linguistics: "Procefedings of the Conference".

Makio Nagao. 1984. A Framowork of a Mechan. ical 'Translation between Japanese and linglish by Analogy Principle. In Alick tilithorn and Ranan Banerji, editors, Artificial and Human Intelligence. IAsevier Science Publishers B.V., Ansterdam, 'The Notherlands.

liernando (. N. Percira. 1985. A StructureSharing Ropresentation for Unification-liased Grammatical lomalisms. In Proceddings of

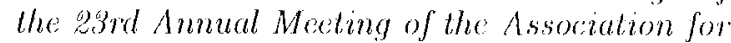
Compulational Linguistics.

Satoshit Sato and Makoto Nagao, 1990. 'Towarel Memory-based 'Lanstation. In COLINC 90 Procectings, Volume 3, pages 247252.

Mike Steel and 'l'andy Warnow. 1993. Kaiokura 'Iree 'l'heorems: Compubing the Maximum Agrecment Subtrec. In Informalion Processintu Letters, 48: 7782. 